\title{
Le libre choix du médecin en milieu hospitalier
}

\author{
B. Burri, président de l'Association suisse des médecins travaillant en cliniques privées et hôpitaux ASMI/SVB
}

L'arrêt du Tribunal fédéral du 31 août 2004 [1] relève que le traitement et le séjour de patients dans les unités de soins privées des hôpitaux justifient des tarifs plus élevés. Cette décision contribue à éclaircir la séparation qui doit être faite entre assurance sociale obligatoire (LAMal) et assurance complémentaire hospitalière (domaines ambulatoire et hospitalier). Des prestations facturées qui dépassent le cadre de l'assurance sociale obligatoire ne sauraient être soumises à la protection tarifaire prévue dans la LAMal. Dans cet esprit, le libre choix du médecin dans un traitement hospitalier est une plus-value de l'assurance complémentaire. L'article qui suit présente un programme en 10 points de l'Association suisse des médecins travaillant en cliniques privées et en hôpitaux (ASMI/SVB), qui définit le complément médical que les médecins agréés apportent aux assurés complémentaires.

Correspondance:

Dr Bernard Burri

Moosstrasse 2

CH-3073 Gümligen-Berne

Tél. 0319527905

Fax 0319527683

E-mail: bernard.burri@bluewin.ch
Depuis l'introduction de la LAMal en 1996, qui sépare sur les plans juridique et fonctionnel, les deux domaines d'assurance maladie que nous connaissons en Suisse, l'Association regroupant les médecins agréés des cliniques et des hôpitaux subventionnés (ASMI) a participé à la définition et aux modes de fonctionnement de l'assurance complémentaire. Dans cette revue déjà, nous avons abordé le complément médical reposant sur 8 axiomes [2]. Par opposition à l'assurance sociale, l'assurance complémentaire, par la volonté du Parlement, a été versée dans le droit privé, accompagnée malheureusement de nombreuses imprécisions que les différents acteurs de la santé ont souhaité corriger à leur convenance. Certaines l'ont été par des décisions des tribunaux, d'autres sont contenues dans la $2^{\mathrm{e}}$ révision de la LAMal - nous pensons en particulier au financement des hôpitaux - dont tout un chacun connaît le devenir incertain. C'est aux confins mal délimités des deux domaines assécurologiques que les conflits sont les plus vifs.

La décision du Tribunal fédéral du 31 août 2004 [1] est un argument éclairant, attendu depuis longtemps, permettant enfin de reconnâ̂tre la ligne de démarcation entre l'assurance sociale et l'assurance complémentaire en cas d'hospitalisation. Le commentaire juridique a déjà été fait à ce propos dans le Bulletin des médecins suisses [3, 4]. Le libre choix du médecin en cas d'hospitalisation n'est pas du domaine de l'assurance sociale mais est le propre de l'assurance complémentaire, tel est l'énoncé central de cet arrêt. Le libre choix du médecin est une plusvalue évidente, toujours selon le TF, s'étendant au-delà de l'assurance obligatoire et par conséquent non soumis à la protection tarifaire. C'est dans le cadre d'un litige entre le département de la santé publique du Canton de Bâle-Ville et un assureur maladie important que la plus haute instance juridique de notre pays s'est prononcée unanimement. L'assureur en question semble avoir encore quelques regrets à posteriori d'avoir participé à cet éclaircissement important [5].

Que représente le libre choix du médecin en cas d'hospitalisation - pour mémoire il est le contenu revendiqué de longue date par les assurances complémentaires - ou mieux dit: que représente-t-il lorsque le patient a fait son choix? Dans le jugement évoqué ci-dessus, le Tribunal fédéral a défini quelques éléments de la plusvalue: le libre choix garantit au patient les soins d'un médecin-chef ou d'un médecin agréé qui effectuera personnellement un certain nombre de gestes habituellement délégués ou encore, des visites plus fréquentes que médicalement indiquées.

Depuis plusieurs années, notre Association s'est attachée à définir avec l'aide de l'Association suisse des patients, de juristes et d'économistes, le complément médical, élément primordial de l'assurance complémentaire, que nous pensons être le réel contenu du libre choix du médecin lors d'un traitement hospitalier. Celuici était étayé par huit axiomes en 2003. Actuellement, notre Association, en tenant compte de l'évolution dans le domaine de la santé, a défini le complément médical en cas d'hospitalisation en 10 points. Ce sont:

1. Le médecin réalise personnellement les prestations médicales.

2. Le médecin a achevé la formation de spécialiste et s'engage dans la formation continue.

3. La disponibilité du médecin est permanente et son accessibilité aisée assure un accompagnement thérapeutique personnel.

4. Le médecin attache une grande importance au souhait du patient dans l'organisation de la prestation.

5. Le médecin met rapidement en œuvre le processus diagnostique ou thérapeutique.

6. En cas d'hospitalisation dans une division privée ou demi-privée, le médecin s'efforce, d'entente avec les établissements privés, d'éviter tout délai d'attente. 
7. Le médecin veille particulièrement à la protection de la sphère privée du patient.

8. Le médecin établit une note d'honoraires transparente et compréhensible.

9. Le médecin accepte les règles de fonctionnement et la commission paritaire, lorsqu'elles existent, des assurances complémentaires.

10. L'éventuel médecin remplaçant, consultant ou mandaté répond à ces mêmes critères.

Les membres de l'ASMI s'engageant, envers leur association, à offrir ce complément médical aux patients ayant souscrit une assurance complémentaire, peuvent en faire état par un certificat nominatif et, s'ils le souhaitent, apparaître sur une liste à disposition des patients sur le site Internet www.asmi.ch. Actuellement, plus de 800 médecins agréés travaillant en cliniques et en hôpitaux ont adhéré à cette démarche et figurent sur la liste à disposition des patients.

Il est indéniable que ces 10 points représentent une astreinte importante pour les médecins

\section{$10^{\mathrm{e}}$ assemblée générale ordinaire ASMI}

Samedi 23 avril 2005, $10.00 \mathrm{~h}$

Hotel Allegro Kursaal, Kornhausstrasse 3, 3013 Bern

A) Partie statutaire (selon invitation)

B) Colloque «Rôle et signification du médecin agréé indépendant travaillant en cliniques privées et hôpitaux publics: Aujourd'hui et demain»

a) vu par le politicien

Dr méd. vét. Markus Dürr, Conseiller d’Etat, Lucerne; président de la Conférence des directeurs sanitaires

b) vu par les assureurs M. Claude Ruey, Conseiller national, Nyon; président Cosama

c) vu par l'économiste

Prof. Dr oec. Günter Neubauer, Institut für Gesundheitsökonomie, Munich

C) Apéritif et dîner

Délai d'inscription: 15 avril 2005 au Secrétariat de l'ASMI agréés mais ils sont l'étoffe que nous avions quelque peu oubliée de l'assurance complémentaire en cas d'hospitalisation. Ce complément à l'assurance sociale, la décision du TF le rappelle, est régi par le droit privé puisqu'il est au-delà de l'assurance sociale et doit être défini financièrement selon les lois de ce domaine et, en particulier, selon celles de la concurrence. Les règles économiques pour la définition du complément médical pourront être établies lorsque le socle, c'est-à-dire la part de l'activité médicale à charge de l'assurance de base, sera déterminé par un tarif. Ce fondement, malgré les réticences que l'on peut parfois rencontrer, doit être intégré dans la réflexion menée sur plusieurs fronts actuellement pour la mise sur pied de forfaits tels les AP-DRG - pour la rémunération des frais hospitaliers.

Dans les considérants de l'arrêt du 31 août 2004, le Tribunal fédéral rappelle un arrêt antérieur où il est clairement dit que la notion de plus-value, qui s'étend donc au-delà de l'assurance obligatoire des soins, concerne également le domaine ambulatoire, contrairement à ce qui se pratique actuellement, puisque la protection tarifaire recouvre tout ce secteur. Les établissements hospitaliers et leurs médecins devraient ainsi repenser, dans cette perspective, le fonctionnement du domaine ambulatoire de l'hôpital.

\section{Références}

1 Arrêt du Tribunal fédéral 5P.74/2004. Décision du 31 août 2004. www.bger.ch/fr/.

2 Burri B. Complément médical en cas d'hospitalisation à charge de l'assurance complémentaire. Bull Méd Suisses 2003;(84)7:298-9.

3 Wanner U. Assurance complémentaire et libre choix du médecin. Bull Méd Suisses 2004; (85) 44:2352-3

4 Kuhn H.P. Le tribunal fédéral arrête le statut des patients privés hospitalisés. Bull Méd Suisses $2004 ;(85) 44: 2338$.

5 Gyger P, Meyer S. Was sagt das Bundesgericht zur freien Arztwahl bei stationären Behandlungen? santésuisse 2005;1-2:20. 